\title{
Event Coverage in Theme Parks Using Wireless Sensor Networks with Mobile Sinks
}

\author{
Gürkan Solmaz and Damla Turgut \\ Department of Electrical Engineering and Computer Science \\ University of Central Florida \\ Email: \{gsolmaz,turgut $\} @$ eecs.ucf.edu
}

\begin{abstract}
Theme parks are large crowded areas with unique characteristics in terms of movement behavior of visitors, attractions in different locations and walking paths connecting the attractions. Wireless sensor networks (WSNs) with mobile sinks can be used for various purposes including security and emergency issues as major challenges in such environments. Modeling of human mobility in theme parks allows us to consider scenario-specific applications of WSNs in these entertainment areas for event coverage purposes.

In this paper, we propose a WSN model with mobile sinks and provide a novel approach to cover the events occurring in the environment. Furthermore, we propose new strategies for mobile sink positioning and event handling decision problems. We evaluate the benefits of our approach through extensive simulations using two sophisticated human mobility models for visitor movement.
\end{abstract}

\section{INTRODUCTION}

Wireless sensor networks usually consist of a set of static sensor nodes and a static sink collecting data from the sensor nodes through hop-by-hop wireless communication. Energy consumption by wireless communications between sensor nodes is a major concern and due to the limited resources of the sensor nodes, decreasing the energy consumption allows maximizing the lifetime of the network. Another problem of the networks with static sensor and sink nodes is adaptivity in dynamic environments. In recent years, variety of research related to WSN applications focus on the use of mobile devices as the elements of the networks. The mobility of nodes in these networks brings new research challenges since the choice of mobility patterns, positioning (placement) of mobile elements and routing protocols significantly affect the performance of the network.

Theme parks are are usually very large, bordered and crowded areas. These environments usually consist of separate various attraction areas distributed in different locations and walking paths connecting these attractions to each other. Visitors are usually walkers without vehicles and the nondeterministic behavior of human movement decisions are combined with the deterministic behavior of the attractions. Realistic modeling of human mobility [1] for such environments allows us to simulate different types of applications and designs of WSNs. Such applications can be used for various purposes including data collection from visitors for commercial purposes, entertainment of customers, crowd management, and so on. Event handling and coverage is one of the major challenges in such environments due to inevitable security and emergency problems.

Almost all the research on network applications in theme parks consider urban sensing applications since most people have smart phones. For instance, mobile applications including multi-player games among the visitors of the park and navigation applications are used for further entertainment. Even though some of these applications are already in use today, none of them considers deploying a WSN application with sensor nodes and mobile sinks without the need of visitor participation. The use of WSN in theme parks may help effective handling of security events such as pick-pocketing by reducing the crime rates and managing emergency situations such as accidents and first-aid.

In this paper, we propose to apply the technology of wireless sensor networks with mobile sinks in the management of a theme park. We tackle the event coverage problem in which the operator needs to acquire the best possible information about the events occurring in the area. The unique characteristics of human mobility and locations of different types of attractions are considered in the model. We evaluate the benefits of the use of WSNs in theme parks by simulations of different scenarios and using two realistic human mobility models for movements of the visitors.

The rest of the paper is organized as follows. We provide a detailed description for our model in Section II. The simulation results are presented in Section III. Section IV summarizes the related work and we conclude in Section V.

\section{Event Coverage}

\section{A. Network model}

The WSN model consists of static sensor nodes randomly distributed and deployed in the theme park and mobile sinks. The sensor nodes have limited energy resources and lifetime while they have the ability to sense an event from their environment and send it to the closest sink via multi-hop transmission at the same time of an event detection.

There are multiple but limited number of mobile sinks with the ability to move very fast to handle the events. We may consider the mobile sinks as expensive but powerful eventhandlers that have the ability to move inside an attraction or between attractions. They collect data from the sensor nodes and share the information about their locations, computation results or event information among each other without any significant time delay. In reality, a mobile sink may be a police officer driving personal electric transportation vehicle (e.g. Segway Patroller [2]) with a powerful computation and wireless communication device such as a tablet computer attached to the vehicle.

The speed of a mobile sink changes dynamically since the environment includes physical constraints such as visitors 


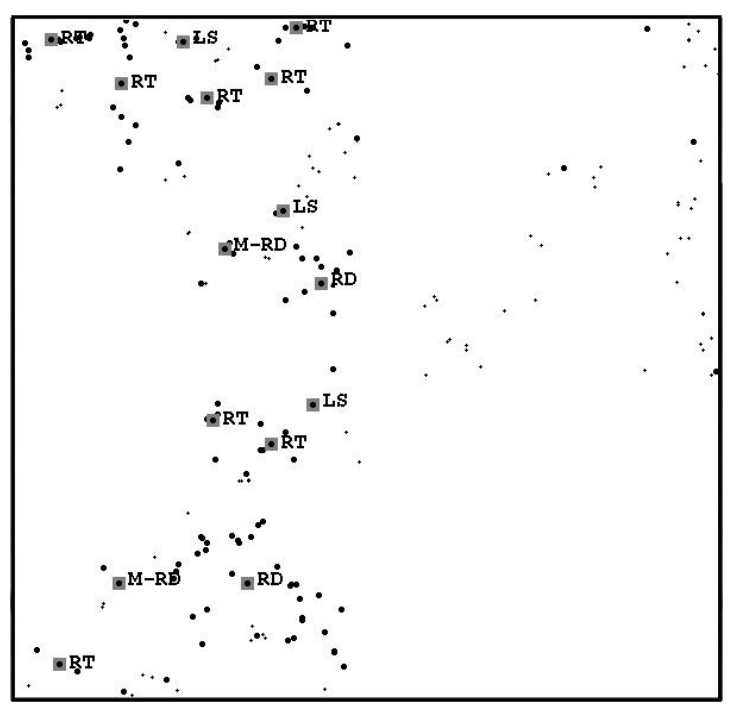

Fig. 1. Queues, non-clustered points and 200 visitors in the theme park.

walking in the same road that a mobile sink needs to move. For synthetic simulations of mobile sink movements, we estimate a speed between two locations for a mobile sink using a simple micro-mobility approach which is explained in the next subsection.

The events occur inside or close to an attraction. We assume that more people in an attraction causes an increase in the possibility of an unwanted event occurring at the attraction. This means crowded areas are more likely to have security events. Each event has a timeout which changes the mode of the event from active to passive. Mobile sinks aim to move towards the event location before the mode of the event becomes passive. We assume that if a mobile sink can move to the location of an event during its active time, the sink handles the event and if the mobile sink is unable to arrive at the event location in a timely manner, the event is considered missed. Each event has a priority value between 1-5 according to the importance of the event. This value is used for adaptive position updates of the mobile sinks.

We use the scenario-specific human mobility model [1] to model attractions and the mobility of the visitors. This mobility model synthetically creates the attractions in a park using different types of queueing models. For instance, restaurants are modeled by $\mathrm{M} / \mathrm{M} / 1$ queues while rides are modeled by $\mathrm{M} / \mathrm{D} / \mathrm{n}$ queues and open-air live shows performed in the park are modeled by $\mathrm{M} / \mathrm{M} / \mathrm{n}$ queues. The queues are created using the fractal points generated as visiting points. DBScan [3] algorithm is used for clustering the visiting points to find the most-populated areas (attractions) people gather to spend time. In Figure 1, the queues generated using the clustered points are represented by squares. The non-clustered visiting points and visitors are shown by small dots and circles respectively. Each queue is presented with its queue type: main rides (RD), medium-sized rides (M-RD), live shows (LS), and restaurants (RT).

\section{B. Dynamic directed graph model}

We propose a dynamic directed graph to model the environment. In this graph model, the nodes represent attractions, mobile sinks and events while the edges are the alternative

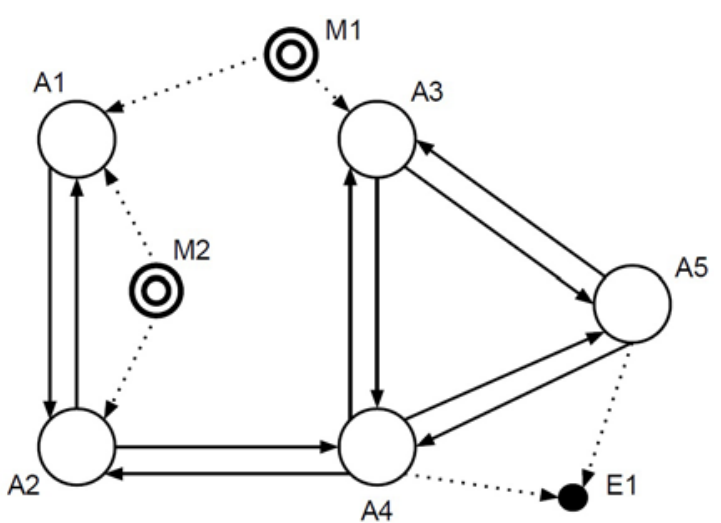

Fig. 2. The directed graph consisting of queues, mobile sinks and an event as nodes.

roads for movement of the mobile sink between the nodes. An example of this graph is illustrated in Figure 2, where $A 1, A 2, \ldots, A n$ are attraction nodes, $M 1, M 2, \ldots, M m$ are mobile sink nodes and $E 1, E 2, \ldots, E e$ are event nodes. Permanent and temporary directed edges are shown by the arrows. Some pair of nodes do not have an edge connecting them, meaning that there is no direct connection or even a need for connection between these nodes. For instance, an edge from an event to a mobile sink or an attraction is not necessary since the goal is to find possible routes for mobile sinks. Attractions have capacities set by a ratio equal to the number of visitors in the attraction divided by the number of all the visitors in the park. These capacities stand for the probability of an event occurring in each of these attractions. In the adaptive approach, the capacities may change by the history of events. Initially, we use weights of the queues for attraction capacities since the number of visitors is proportional to these weights. The total capacity (probability) of all attractions is equal to 1 .

The edges of the graph are weighted and directed. The weights of the edges are the time estimates for mobile sink to travel along this edge from the source node to the target node. The estimated time for an edge is computed by Algorithm 1 . In this algorithm, we assume the number and directions of visitors, distance and width of a road effect the moving time of a mobile sink. This assumption is based on the fact that if the density of people is high then the number of collisions avoided by slowing down along the road for the mobile sink is also high. The edge weights change dynamically and updated according to the current movement patterns and positions of visitors in the park. $c$ is the density calculated by the total area $a$ of visitors currently located in the road divided by the area of the road. The area of the road is equal to $d(s, t) \cdot w$ where $w$ is the width. $\alpha$ is the direction difference between the sink and a visitor and $\gamma \epsilon(0,1]$ is the constant for adjusting the effect of the direction $\alpha$. For instance, the effect of collision detection is highest when a visitor moves to the opposite direction and lowest if they are moving in the same direction. $\beta$ is a constant parameter which configures the magnitude of the speed change. We used $12.5 \mathrm{mph}$ as the maximum mobile sink speed as it is listed in the Segway Patroller specification sheet [2] for the models $x 2$ and i2. Today, more than 1,200 police departments and public safety organizations in the world use these devices for security purposes. 


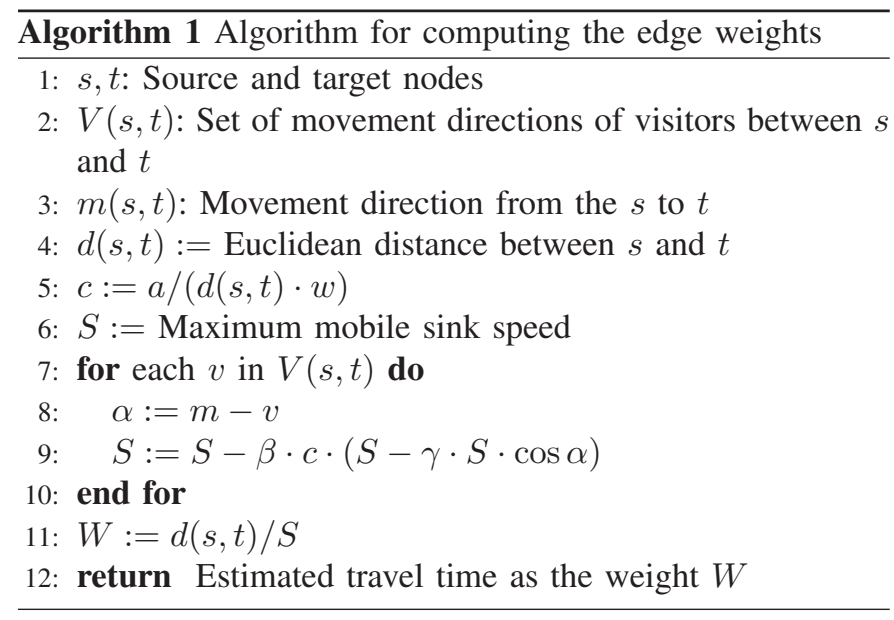

\section{Event coverage strategies}

The problem of the event coverage is the capability of the mobile sink reaching an event sensed by sensor nodes in minimal time. To achieve this goal, we first need to position the mobile sinks in the theme park. Then, we need to select the best path and the best mobile sink for all events.

For the problem of positioning mobile sinks in the distinct attractions, we first propose a weighted sink positioning strategy for the multiple mobile sinks. In this strategy, we assume that the number of visitors is proportional to the number of events occurring in the attraction. By monitoring the approximate number of visitors of all attractions, we assign the capacity values to attractions as the probability of having events and update their values iteratively at discrete update times. The mobile sinks are distributed to the attractions according to the current capacities of the attractions.

For some scenarios, there may be a subset of attractions having higher rates of events compared to the other attractions and these rates may also change in time. This behavior seems probable since the theme park environments are dynamic and likely to have unexpected changes.

As a result, we propose another strategy called, the adaptive sink positioning. In this approach, sinks are distributed according to attraction capacities in the same fashion, but this time the mobile sinks keep history of all events happened by sharing the event data among each other. The data includes the index of the closest attraction node and priority of the event. After occurring of a new event, the capacity of the closest attraction increases by a value that is the multiplication of a constant value $\delta$ (e.g. $\delta:=0.05$ ) and priority of the event, while the capacities of other attractions decrease by the same value divided by the total number of the other attractions. The sinks have common location update times. Each of the sinks decide and move to the next attraction at the update times according to the current capacities, occupancy by the other sinks and existence of an edge between the current and the next attraction.

For effective event handling, we apply shortest path algorithm by using the directed graph with values updated according to the current edge weights for each mobile sinks. In this Shortest Path (SP) strategy, each mobile sink finds its shortest path to the event in terms of travel times when an event occurs and the mobile sinks share their estimated travel times among themselves. The mobile sink with the minimum estimated travel time is selected as the best sink to handle the event. When the chosen sink move to an event area, other sinks update their positions according to the new unoccupied attraction left by the selected sink.

For the environments in which monitoring movements of visitors is not possible, we propose the Closest Sink (CS) strategy. In this strategy, the shortest path algorithm is used in the same fashion as in the SP strategy, but edge weights are static. The weights of the edges are calculated by the Euclidean distances between the attractions.

\section{Simulation Study}

\section{A. Simulation environment and metrics}

We use theme park (TP) [1] and SLAW [4] mobility models to model the human movements. Using the synthetic simulations of the two models, trajectory data for mobility of visitors in the theme park is integrated into our simulation of WSN with mobile sinks. We used the same fractal point files including data for 1000 points distributed in the terrain of the same size in order to compare the performances with these two mobility models.

The simulation environment consists of 15 attractions distributed as the directed graph attraction nodes and one or multiple (up to 10) mobile sink nodes. Edges between the attraction nodes are created initially in two ways, one is having edge between the closest attractions with a vertex degree of 4 and the other is creating edges randomly between the attractions with the graph density of 0.7 . Temporary edges for the mobile sinks and the events are created to the closest attraction. Event nodes are created temporarily and distributed in three ways: uniformly random selection, selection according to the weights and selection from a number of randomly selected specific attractions. In figures with simulation runs, each simulation run represents an average result of a set of 20 experiments with the same selected attractions to hold events. The attractions having events vary for different simulation runs due to the event attraction selection schemes.

Location update time for adaptive mobile sinks is $30 \mathrm{~min}$ utes and starting times of 300 events are distributed to the total simulation time by uniform random distribution. For the events, active times are generated randomly between the minimum of 60 seconds and the maximum of 300 seconds. Table I summarizes the other parameters used in our simulation study.

TABLE I

SIMULATION PARAMETERS

\begin{tabular}{|l|l|}
\hline simulation time & 10 hours \\
\hline terrain size & $1000 \times 1000 \mathrm{~m}$ \\
\hline number of visitors & 500 \\
\hline speed of visitors & $1.00 \mathrm{~m} / \mathrm{sec}$ \\
\hline max mobile sink speed & $5.58 \mathrm{~m} / \mathrm{sec}$ \\
\hline$\delta$ for adaptive updates & 0.1 \\
\hline sampling time of visitors & $10 \mathrm{sec}$ \\
\hline
\end{tabular}

The simulation metrics used in simulations are average event handling time, that is the travel time for the selected mobile sink to reach to an event after it occurs, and hit ratio, that is the success rate of mobile sinks calculated by dividing the number of hits by the total number of events occurred. 


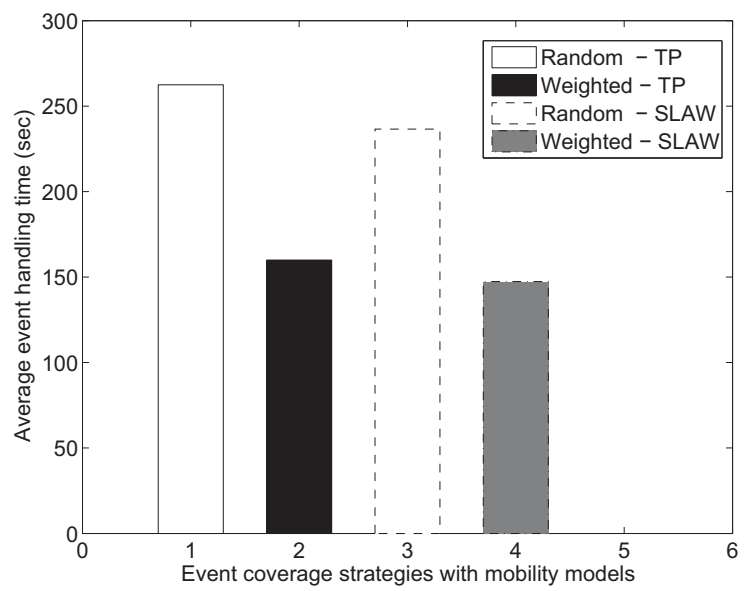

Fig. 3. Average event handling times: Weighted vs. Random sink positioning.

\section{B. Simulation results}

1) Weighted sink positioning: In the first of set of experiments, we evaluated the performance of the weighted sink positioning strategy. We compared the performance of this strategy against the random sink positioning. In the random positioning, the mobile sinks are distributed to the attractions by uniformly random selection of one attraction for each one of the sinks. The number of mobile sinks is 5 and events are created according to the distribution of visitors in attractions. The shortest path (SP) strategy is used for event handling of the mobile sinks.

Figure 3 shows the average event handling times for weighted and random sink positioning for TP and SLAW mobility models. For both mobility models, the weighted sink positioning is a clear winner compared to the random positioning since the sinks need less time to handle an event.

2) Adaptive sink positioning: Next, we evaluated the success of adaptive positioning of mobile sinks in the case when events consistently occur specifically from a subset of attractions. We compared the adaptive sink positioning against the weighted sink positioning and observed the positive effects of adaptive positioning on event handling capabilities. The number of sinks is 3 and 3 attractions are selected uniformly random from all attractions as specific attractions where events occur. As in the previous experiments, the SP strategy is used for event handling of the mobile sinks.

Figure 4 shows that there is a significant difference for the average event handling times, comparing the results of the adaptive and weighted sink positioning strategies. For each simulation run, adaptive positioning produces results more or less stable and successful average times from 70 seconds to 90 seconds, while the results of weighted positioning fluctuate between 160 seconds and 360 seconds. These are expected behavior of both strategies, since in adaptive positioning, no matter where the sinks are located initially, they are able to move to the attractions where events consistently occur. On the other hand, in weighted positioning, the sinks do not change their allocation of attraction according to the past events. For instance, in one simulation run, events may occur close to the initially heavy weighted attractions but in another

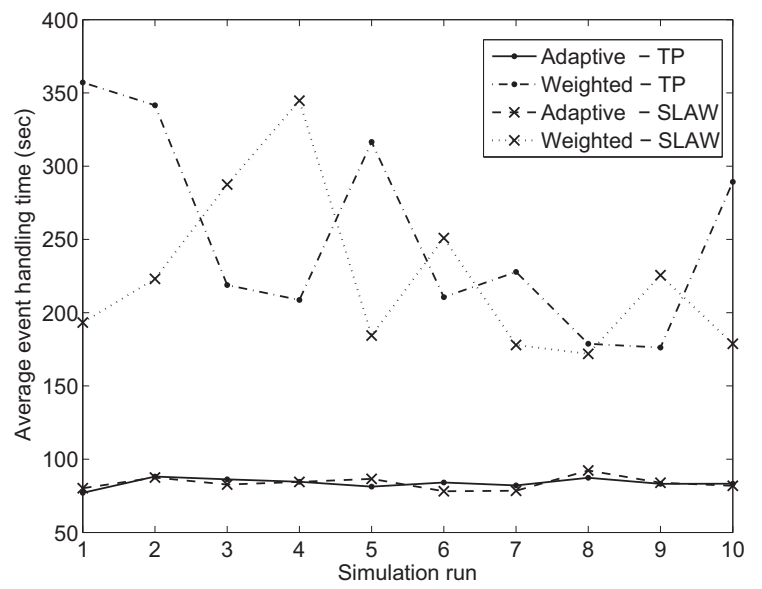

Fig. 4. Average event handling times: Adaptive vs. Weighted sink positioning.

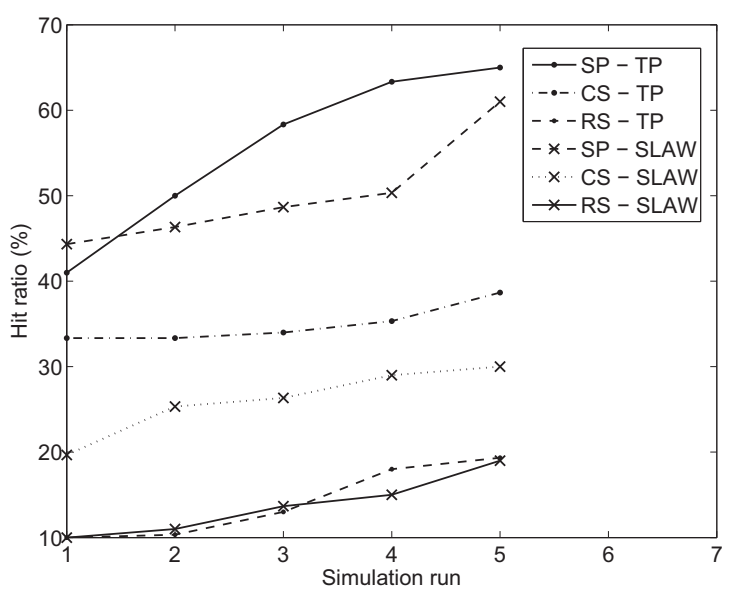

Fig. 5. Hit ratios w.r.t. the event handling strategies: Shortest Path (SP), Closest Sink (CS), and Random Sink (RS).

simulation run, events may occur close to the attractions with low capacities. Although events occur in different attractions, sinks are always located close to the attractions with high capacities for the weighted positioning strategy.

3) Event handling decision: We evaluated the performance of generation of shortest path strategy using the dynamic edge weights for each mobile sink. For this reason, we compared the Shortest Path (SP) event handling strategy with Closest Sink (CS) strategy in which we generate shortest paths for each mobile sink using the distances between the attractions as edge weights if there is an edge in the directed graph and the Random Sink (RS) strategy in which we select one of the mobile sinks randomly and send the selected sink according to its shortest path. We used weighted positioning with 3 mobile sinks and events are distributed randomly according to the attraction capacities in all experiments.

As shown in Figure 5, SP strategy has better results in terms of hit ratios and CS strategy produces significantly better results compared to RS strategy. Although results may differ in different simulation runs, SP strategy always has the significant advantage over the other two strategies. 


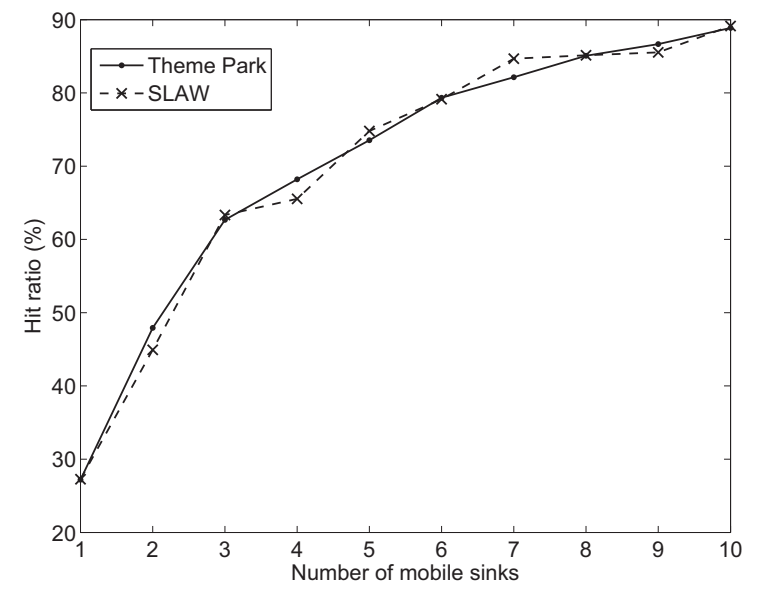

Fig. 6. Effect of multiple mobile sinks on hit ratio.

4) Use of multiple mobile sinks: Lastly, we compare the use of different numbers of mobile sinks in the network. Since sinks are expensive elements of the network, we expect to reach a convergence point for the results of the hit ratios.

As in Figure 6, using two or three mobile sinks produced better hit ratios compared to the use of single mobile sink. On the other hand, after a certain point, the effect of additional sinks starts losing its significance. For instance, using no more than 7 mobile sinks seems reasonable since the impact of the 3 extra sinks is not significant. By looking at the hit ratios of single mobile sink and multiple mobile sinks, it is clear that using multiple mobile sinks increased the success rate of the event handling from values below $30 \%$ to the values close to $90 \%$. This is the main reason why we propose the WSN model with multiple mobile sinks for the theme park scenario.

\section{RELATED WORK}

For managing positioning and mobility of the sink nodes, various approaches are proposed in the literature. Mulligan and Ammari [5] provides an extensive survey on coverage in WSN. Younis and Akkaya [6] survey techniques for node placement strategies used in WSN. While Vincze et al. [7] use an approach, similar to our weighted positioning strategy, for positioning multiple sinks optimally in a sensor network based on an electrostatic model by assigning positive or negative charges to sensor nodes, and positive charges to sinks. In this study, the goal is to optimize energy usage of sensor nodes in the network. Melodia et al. [8] suggest location management scheme for the mobility of actors based on a hybrid strategy including location updates and location prediction. Akbas et al. [9] propose the actor positioning algorithm utilizing the Valence Shell Electron Pair Repulsion (VSEPR) theory of chemistry, which is based on the correlation between molecular geometry and the number of atoms in a molecule. Akbas et al. [10] have eliminated the limitations of the basic VSEPR theory by extending the approach for multiple central data collectors. Vincze et al. [11] introduce an adaptive approach for sink mobility in an event-driven multi-hop WSN to minimize the maximum load on sensors and prolong the lifetime of the network. The intruder movement model is used as the event model. They propose to minimize both the sum of event distances and the maximum energy consumption. For transmission scheduling problem, Turgut and Bölöni [12] [13] first describe a graph-theory based approach for calculating the optimal policy and compare three heuristics based on different principles (imitation of human decision making, stochastic transmission and constant risk) to control the transmission behavior of the nodes in the presence of multiple mobile sinks in WSN. Bölöni and Turgut [14] suggest a decision-theoretic approach. They first develop a dynamic programming based optimal algorithm for the case when the mobility of the sinks is known in advance and then propose two decision theoretic algorithms which use only probabilistic models learned from the history of interaction with the mobile sinks, and do not require knowledge about their future mobility patterns. Wang et al. [15] survey movement strategies for improving the performance of event coverage in wireless sensor networks.

\section{COnClusion}

In this paper, we tackled the event coverage problem in theme parks and proposed new strategies for event handling and sink positioning. The success of our approaches are evaluated through extensive simulations of different scenarios using the scenario-specific theme park (TP) and SLAW mobility models.

\section{REFERENCES}

[1] G. Solmaz, M. Akbas, and D. Turgut, "Modeling visitor movement in theme parks," in Proc. of the IEEE LCN'12, October 2012, pp. 36-45.

[2] "SEGWAY," http://www.segway.com.

[3] M. Ester, H. P. Kriegel, J. Sander, and X. Xu, "A density-based algorithm for discovering clusters in large spatial databases with noise," in Proc. of the ICKDDM'96, August 1996, pp. 226-231.

[4] K. Lee, S. Hong, S. J. Kim, I. Rhee, and S. Chong, "SLAW: self-similar least-action human walk," IEEE/ACM Transactions on Networking, vol. 20, no. 2, pp. 515-529, April 2012.

[5] R. Mulligan and H. M. Ammari, "Coverage in wireless sensor networks: A survey," Journal of Network Protocols and Algorithms (NPA), vol. 2, no. 2, pp. 27-53, June 2010.

[6] M. Younis and K. Akkaya, "Strategies and techniques for node placement in wireless sensor networks: a survey," Ad Hoc Networks, vol. 6 , no. 4, pp. 621-655, June 2008.

[7] Z. Vincze, K. Fodor, R. Vida, and A. Vidács, "Electrostatic modelling of multiple mobile sinks in wireless sensor networks," in Proc. of the IFIP Networking Workshop on Performance Control in Wireless Sensor Networks, May 2006, pp. 30-37.

[8] T. Melodia, D. Pompili, and I. F. Akyildiz, "Handling mobility in wireless sensor and actor networks," IEEE Transactions on Mobile Computing, vol. 9, no. 2, pp. 160-173, February 2010.

[9] M. Akbas and D. Turgut, "APAWSAN: Actor positioning for aerial wireless sensor and actor networks," in Proceedings of IEEE LCN'11, October 2011, pp. 567-574.

[10] M. Akbas, G. Solmaz, and D. Turgut, "Actor positioning based on molecular geometry in aerial sensor networks," in Proc. of the IEEE ICC'12, June 2012, pp. 513-517.

[11] V. Zoltán, V. Dorottya, V. Rolland, V. Attila, and T. András, "Adaptive sink mobility in event-driven multi-hop wireless sensor networks," in Proc. of the InterSense'06, May 2006.

[12] D. Turgut and L. Bölöni, "Heuristic approaches for transmission scheduling in sensor networks with multiple mobile sinks," The Computer Journal, vol. 54, no. 3, pp. 332-344, March 2011.

[13] D. Turgut and L. Bölöni, "Three heuristics for transmission scheduling in sensor networks with multiple mobile sinks," in Proc. of the AAMAS'08 Workshop on Agent Technology for Sensor Networks, May 2008, pp. $1-8$.

[14] L. Bölöni and D. Turgut, "Should I send now or send later? A decisiontheoretic approach to transmission scheduling in sensor networks with mobile sinks," Wireless Communications and Mobile Computing Journal, vol. 8, no. 3, pp. 385-403, March 2008.

[15] B. Wang, H. B. Lim, and D. Ma, "A survey of movement strategies for improving network coverage in wireless sensor networks," Computer Communications, vol. 32, no. 13-14, pp. 1427-1436, August 2009. 